\title{
Radiofrequency ablation of benign thyroid nodules: recommendations from the Asian Conference on Tumor Ablation Task Force
}

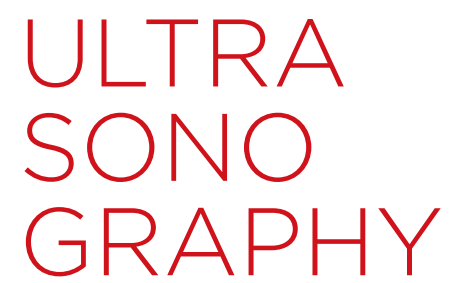

\author{
Eun Ju Ha ${ }^{1}$, Jung Hwan Baek², Ying Che ${ }^{3}$, Yi-Hong Chou ${ }^{4,5,6}$, Nobuhiro Fukunari ${ }^{7}$, \\ Ji-hoon $\mathrm{Kim}^{8}{ }^{8}$, Wei-Che Lin ${ }^{9}$, Le Thi My ${ }^{10}$, Dong Gyu Na ${ }^{11}$, Lawrence Han Hwee Quek ${ }^{12}$, \\ Ming-Hsun $\mathrm{Wu}^{13}$, Koichiro Yamakado ${ }^{14}$, Jianhua Zhou $^{15}$
}

'Department of Radiology, Ajou University School of Medicine, Suwon; ${ }^{2}$ Department of Radiology and Research Institute of Radiology, Asan Medical Center, University of Ulsan College of Medicine, Seoul, Korea; ${ }^{3}$ Department of Ultrasound, the First Affiliated Hospital of Dalian Medical University, Dalian, China; ${ }^{4}$ Department of Medical Imaging and Radiological Technology, Yuanpei University of Medical Technology, Hsinchu; ${ }^{5}$ Department of Radiology, Taipei Veterans General Hospital and School of Medicine, National Yang Ming University, Taipei; ${ }^{6}$ Department of Radiology, Yee Zen General Hospital, Taoyuan, Taiwan; ${ }^{7}$ Department of Surgery, Showa University School of Medicine, Northern Yokohama Hospital, Yokohama, Japan; ${ }^{8}$ Department of Radiology, Seoul National University Hospital, Seoul, Korea; ${ }^{9}$ Department of Diagnostic Radiology, Kaohsiung Chang Gung Memorial Hospital and Chang Gung University College of Medicine, Kaohsiung, Taiwan; ${ }^{10}$ Department of Radiology, Vinmec Times City International Hospital, Hanoi, Vietnam; ${ }^{11}$ Department of Radiology, GangNeung Asan Hospital, Gangneung, Korea; ${ }^{12}$ Department of Diagnostic Radiology, Tan Tock Seng Hospital, Singapore; ${ }^{13}$ Department of Surgery, National Taiwan University Hospital, Taipei, Taiwan; ${ }^{14}$ Department of Radiology, Hyogo College of Medicine, Nishinomiya, Japan; ${ }^{15}$ Department of Ultrasound, Sun Yat-Sen University Cancer Center, State Key Laboratory of Oncology in South China, Collaborative Innovation Center for Cancer Medicine, Guangzhou, China

Radiofrequency ablation (RFA) is a thermal ablation technique widely used for the management of benign thyroid nodules. To date, five academic societies in various countries have reported clinical practice guidelines, opinion statements, or recommendations regarding the use of thyroid RFA. However, despite some similarities, there are also differences among the guidelines, and a consensus is required regarding safe and effective treatment in Asian countries. Therefore, a task force was organized by the guideline committee of the Asian Conference on Tumor Ablation with the goal of devising recommendations for the clinical use of thyroid RFA. The recommendations in this article are based on a comprehensive analysis of the current literature and the consensus opinion of the task force members.

Keywords: Thyroid; Ultrasonography; Radiofrequency ablation; Guidelines

\section{REVIEW ARTICLE}

https://doi.org/10.14366/usg.20112 pISSN: 2288-5919 • elSSN: 2288-5943

Ultrasonography 2021;40:75-82

Received: July 24, 2020

Revised: September 8, 2020

Accepted: September 8, 2020

Correspondence to:

Jung Hwan Baek, MD, PhD,

Department of Radiology and Research Institute of Radiology, Asan Medical Center, University of Ulsan College of Medicine, 88 Olympic-ro 43-gil, Songpa-gu, Seoul 05505, Korea

Tel. +82-31-219-4057

Fax. +82-31-219-5852

E-mail: radbaek@naver.com

This article will be secondarily published in 2021 April-June issue (Vol 29, No 2) of Journal of Medical Ultrasound (the official journal of Asian Federation of Societies for Ultrasound in Medicine).

This is an Open Access article distributed under the terms of the Creative Commons Attribution NonCommercial License (http://creativecommons.org/ licenses/by-nc/4.0/) which permits unrestricted noncommercial use, distribution, and reproduction in any medium, provided the original work is properly cited.

Copyright (C) 2021 Korean Society of Ultrasound in Medicine (KSUM)

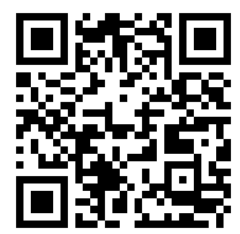

How to cite this article:

Ha EJ, Baek JH, Che Y, Chou YH, Fukunari $\mathrm{N}$, Kim JH, et al. Radiofrequency ablation of benign thyroid nodules: recommendations from the Asian Conference on Tumor Ablation Task Force. Ultrasonography. 2021 Jan;40(1):75-82. 


\section{Introduction}

Ultrasound (US)-guided radiofrequency ablation (RFA) is an alternative to surgery for patients with benign thyroid nodules [17]. Since 2001, many studies, including randomized controlled trials and meta-analyses, have reported the effectiveness and safety of this approach, resulting in its widespread use in patients with benign thyroid nodules [8-12]. Since the first recommendations of the Korean Society of Thyroid Radiology (KSThR) in 2009, academic societies in Italy, the United Kingdom, and Austria have established their own clinical practice guidelines, opinion statements, or recommendations for thyroid RFA [3-6]. Currently, the revised 2017 guidelines of the KSThR serve as the standard [2].

Despite some similarities, there are also differences among the clinical practice guidelines $[2-6,13,14]$. In addition, controversies exist that may be confusing for clinicians performing RFA [13]. Therefore, the guideline committee of the Asian Conference on Tumor Ablation (ACTA) organized a task force to devise recommendations for the use of RFA in the management of benign thyroid nodules. A systematic search of international databases, including MEDLINE and Embase, was performed using the keywords "thyroid" AND ("radiofrequency ablation" OR "RF ablation" OR "RFA") AND ("guideline" OR "recommendation" OR "opinion" OR "statement"). The search was limited to studies published in English between January 1, 2000 and August 2, 2019. Studies or subsets of studies that satisfied the following criteria were included: (1) population and intervention: benign thyroid nodules and image-guided RFA; (2) study design: clinical practice guidelines (opinion statement, recommendations, or guidelines); (3) outcomes: indications, preprocedural and postprocedural evaluation techniques and informed consent. In addition, an up-to-date search was conducted with the aim of answering some key questions. Based on a comprehensive analysis of the current literature, we generated online communications. Tables 1-3 describe the scope of the guidelines, methodology, and general information. The purpose of the recommendations is to provide a consensus expert opinion, based on the best scientific evidence available, to guide members of the ACTA in the use of thyroid RFA for the safe and effective management of benign thyroid nodules.

\section{Methodology}

Following a review of the existing literature, a grading system was established based on the quality of evidence, clinical benefits and harms, costs, and patient preferences [15-17]. The modified Delphi method was used to formulate the recommendations, particularly regarding benefits and harms [17].
The committee comprised 13 experts in thyroid interventions, collectively representing six Asian countries. The level of evidence and benefit to patients were the main factors underlying the recommendations. The clinical applicability was discussed, as well as

Table 1. Scope of the guidelines

\begin{tabular}{ll}
\hline \multicolumn{1}{c}{ Scope of the guidelines } & \multicolumn{1}{c}{ Comment } \\
\hline $\begin{array}{l}\text { Disease/condition } \\
\text { Guideline category } \\
\text { Clinical specialty }\end{array}$ & $\begin{array}{l}\text { Benign thyroid nodules } \\
\text { Ultrasound-guided RFA } \\
\text { Thyroid specialists (radiologists, } \\
\text { endocrinologists, surgeons, and other } \\
\text { thyroid specialists) } \\
\text { To evaluate the use of RFA for patients } \\
\text { with benign thyroid nodules }\end{array}$ \\
Guideline objective & $\begin{array}{l}\text { Patients with benign thyroid nodules } \\
\text { Target population }\end{array}$ \\
Interventions & $\begin{array}{l}\text { RFA } \\
\text { Major outcomes considered }\end{array}$ \\
& $\begin{array}{l}\text { Utility of RFA for treating benign thyroid } \\
\text { nodules and decision-making issues }\end{array}$ \\
\hline
\end{tabular}

RFA, radiofrequency ablation.

Table 2. Methodology

\begin{tabular}{cl}
\hline Methodology of guidelines & \multicolumn{1}{c}{ Comment } \\
\hline Method used to collect data & $\begin{array}{l}\text { Searches of electronic databases, including } \\
\text { Ovid-MEDLINE }\end{array}$ \\
Literature search procedure & The MEDLINE literature search considered \\
& English-language articles published \\
between January 1, 2000 and August & 2,2019, using the following keywords: \\
& ("thyroid" AND ["radiofrequency ablation" \\
& OR "RF ablation" OR "RFA"] AND ["guideline" \\
& OR "recommendation" OR "opinion" OR \\
& "statement"]) \\
& Modified Delphi method \\
Method used to formulate \\
recommendations
\end{tabular}

Table 3. General information

\begin{tabular}{|c|c|}
\hline General information & Comment \\
\hline Source of funding & None \\
\hline $\begin{array}{l}\text { Guideline committee } \\
\text { members }\end{array}$ & $\begin{array}{l}\text { Chair: Jung Hwan Baek } \\
\text { Secretary: Eun Ju Ha } \\
\text { China: Ying Che/Jianhua Zhou } \\
\text { Japan: Nobuhiro Fukunari/Koichiro Yamakado } \\
\text { Taiwan: Wei-Che Lin/Yi-Hong Chou/Ming-Hsun Wu } \\
\text { Korea: Ji-hoon Kim/Dong Gyu Na } \\
\text { Singapore: Lawrence Han Hwee Quek } \\
\text { Vietnam: Le Thi My }\end{array}$ \\
\hline $\begin{array}{l}\text { Financial disclosures/ } \\
\text { conflicts of interest }\end{array}$ & $\begin{array}{l}\text { Dr. Baek has acted as a consultant for two } \\
\text { companies, STARmed and RF Medical, since } 2017 . \\
\text { No other member of the guideline committee has } \\
\text { any financial disclosure or conflict of interests to } \\
\text { declare. }\end{array}$ \\
\hline
\end{tabular}


the impact of the recommendations on patient outcomes. Despite a high Delphi score, the recommendations were downgraded based on the consensus opinion of the committee.

\section{Indications}

Key question 1: What are the indications for RFA of benign, nonfunctioning thyroid nodules?

Recommendation 1

RFA is indicated for patients with benign, nonfunctioning thyroid nodules with symptoms or cosmetic issues.

For benign, nonfunctioning thyroid nodules, RFA should be restricted to patients with symptoms or cosmetic issues. Although the majority of benign thyroid nodules are asymptomatic and treatment is generally not required, some patients require treatment due to symptoms such as dysphagia, throat discomfort, foreign body sensation, pain, anterior neck mass, and cough, as well as for cosmetic reasons. Therefore, the purpose of RFA for benign, nonfunctioning thyroid nodules is to relieve symptoms and address cosmetic issues. A symptom score of $0-10$ is generated using a 10-cm visual analog scale. A cosmetic score of 1-4 is derived by a physician, as follows: 1, no palpable mass; 2, no cosmetic issues but a palpable mass; 3 , cosmetic issue only during swallowing; 4, nodule visible to the naked eye.

\section{Debate and consensus}

No definitive cutoff values are available for the size of benign thyroid nodules suitable for RFA. Most guidelines emphasize the importance of the patient's symptoms and cosmetic concerns when making a clinical decision regarding RFA. Although large thyroid nodules often cause symptoms and cosmetic issues, the patient's neck circumference and the location of the thyroid nodules are critical factors. The KSThR guidelines recommend that RFA can be considered for growing nodules $>2.0 \mathrm{~cm}$ in size [2]. Italian guidelines also recommend RFA for nodules $>2.0 \mathrm{~cm}$ in size $[3,4]$. Although there are no absolute contraindications for RFA, thyroid nodules showing retrosternal extension or extension into the Zuckerkandl tubercle can be difficult to access and multiple treatment sessions may be required. The use of a bipolar electrode can be recommended for pregnant women or patients with electrical devices, such as a cardiac pacemaker [18-20].

Key question 2: What are the indications for RFA in patients with autonomously functioning thyroid nodules (AFTNs)? Recommendation 2

RFA may be indicated for AFTNs that are either toxic or pretoxic.

Although radioactive iodine (RAI) therapy and surgery are effective and relatively safe treatment options, hypothyroidism after surgery and RAI therapy may exacerbate pre-existing chronic conditions in the elderly and its use is also controversial in young women. Moreover, some patients refuse RAl therapy or surgery because of concerns regarding radiation exposure and potential complications, such as hypothyroidism. RFA can serve as an alternative treatment option for patients who refuse, or cannot undergo, traditional treatments, such as RAI or surgery, as well as for patients with a favorable condition, such as a small single AFTN $[11,21-23]$.

\section{Debate and consensus}

Regarding the size of AFTNs considered suitable for RFA, all guidelines agree that small AFTNs can be effectively treated by a single session of RFA, with preservation of normal thyroid tissue function. However, some guidelines suggest that RFA may be less effective for large (volume $>15-20 \mathrm{~mL}$ ) or multifocal ATFNs [3-5]. The Italian guidelines recommended combined RFA and RAI treatment for large ATFNs (volume $>20 \mathrm{~mL}$ ) for more rapid improvement of local symptoms $[3,4]$. Recent meta-analyses have suggested that thermal ablation of AFTNs is an option regardless of nodule size. However, treatment efficacy may be lower in larger nodules and multiple sessions of RFA may be necessary $[11,24]$. Based on thyroid-stimulating hormone (TSH) normalization, no significant difference in the treatment response rate was seen between nodules with a volume $\leq 18$ and $>18 \mathrm{~mL}(73.6 \%$ vs. $67.0 \%$, respectively; $P=0.53$ ) [24]. Regarding the number of treatment sessions, single and multiple sessions of RFA achieved TSH normalization in $66.7 \%$ and $79.3 \%$ of cases, respectively ( $P=0.23$ ) [24]. The majority of the ACTA committee members agreed that RFA could be indicated for AFTNs regardless of nodule size. However, if clinical success (defined as TSH normalization) is likely to be difficult to achieve with one session (e.g., in cases of large nodule size, multifocality, or poor localization), RAI therapy with or without RFA or surgery may be considered first, instead of RFA. Multiple RFA sessions could be an option if patients refuse or cannot undergo surgery or RAl therapy in these situations. This issue should be evaluated further in future studies.

\section{Preprocedural Evaluation}

Key question 3: How can we confirm that thyroid nodules are benign before the procedure?

\section{Recommendation 3.1}

Thyroid nodules should be confirmed as benign on the basis of at least two US-guided fine-needle aspirations (FNAs) or core needle biopsies (CNBs) prior to RFA.

Before the procedure, thyroid nodules should be confirmed as 
benign on at least two FNAs or CNBs. Two benign biopsy results are required to prevent a possible false-negative diagnosis of malignancy (typically follicular carcinoma or follicular variant papillary thyroid carcinoma). However, even if thyroid nodules are confirmed to be benign, RFA should be performed carefully when there are suspicious US features.

\section{Recommendation 3.2}

A benign diagnosis based on a single FNA or CNB is sufficient when the nodule has US features highly suggestive of benign status (spongiform or partially cystic nodules with an intracystic comet tail artifact) and for AFTNs.

\section{Debates and consensus}

According to the KSThR guidelines, a single benign diagnosis is sufficient for thyroid nodules with US features highly suggestive of benign nodules (isoechoic spongiform or partially cystic nodules with an intracystic comet tail artifact) and for AFTNs [2]. Similarly, the Italian guidelines accept a single benign diagnosis for thyroid nodules shown by US to be entirely spongiform or ovoid, smooth, and isoechoic/hyperechoic, and for AFTNs [3,4]. Although USbased risk stratification systems differ among countries, we consider a single benign biopsy result to be acceptable for RFA of American College of Radiology (ACR) TR-1 nodules (spongiform), European Thyroid Imaging Reporting and Data System (EUTIRADS) category 2 nodules (spongiform), American Association of Clinical Endocrinologists/American College of Endocrinology and Associazione Medici Endocrinologi (AACE/ACE-AME) low-risk nodules (spongiform and mostly cystic, with intracystic comet tail artifacts), and KSThR Thyroid Imaging Reporting and Data System (K-TIRADS) category 2 (spongiform or partially cystic with intracystic comet tail artifacts) nodules, given the very low risk of malignancy (typically <1\%) [25-28]. However, although they are almost always benign when there is no co-occurring suspicious US feature for malignancy, a cautious approach is necessary to reduce the possibility of a false-negative diagnosis. On the contrary, follicular and follicular variant papillary thyroid carcinomas often show US features corresponding to ACR TR-2 (partially cystic isoechoic nodules without suspicious US features), EU-TIRADS category 3 (entirely isohyperechoic), AACE/ACE-AME intermediate-risk, American Thyroid Association Thyroid Imaging, Reporting and Data System very low-risk (partially cystic without suspicious US features), or K-TIRADS category 3 (isoechoic or partially cystic without suspicious US features) nodules [15,25-28]. Therefore, at least two benign biopsy results are necessary for these nodules before considering RFA, even though the risk may be low (approximately $2 \%-5 \%$ ). Nodule selection for RFA should be decided based on a consensus between the patient and the clinician, who can evaluate the therapeutic options based on the patient's best interests.

Table 4 lists the evaluations performed prior to RFA of benign thyroid nodules. The US features of thyroid nodules and patients' symptom/cosmetic scores should be carefully considered. Nodule size, echogenicity, margin, solid component (\%), internal vascularity and cervical lymph nodes should be evaluated in detail by US. The orthogonal diameter should be measured three times; the volume of the thyroid nodule should also be measured at baseline. Laboratory tests should include a complete blood count, coagulation test (bleeding, prothrombin, and activated partial thromboplastin times), and thyroid function test. The thyroid function test should include at least thyrotropin, triiodothyronine (T3), and free thyroxine (fT4). Hypothyroidism should be addressed by hormone replacement therapy, and hyperthyroidism should be further evaluated by a technetium ${ }^{99 \mathrm{~m}} \mathrm{Tc}$ pertechnetate or ${ }^{123} \mathrm{I}$ thyroid scan. A thyroid function test is important to evaluate the treatment outcome of patients with AFTNs, where remission or normalization of

\section{Table 4. Preprocedural checklist for radiofrequency ablation of} benign thyroid nodules

The evaluations prior to RFA of benign thyroid nodules

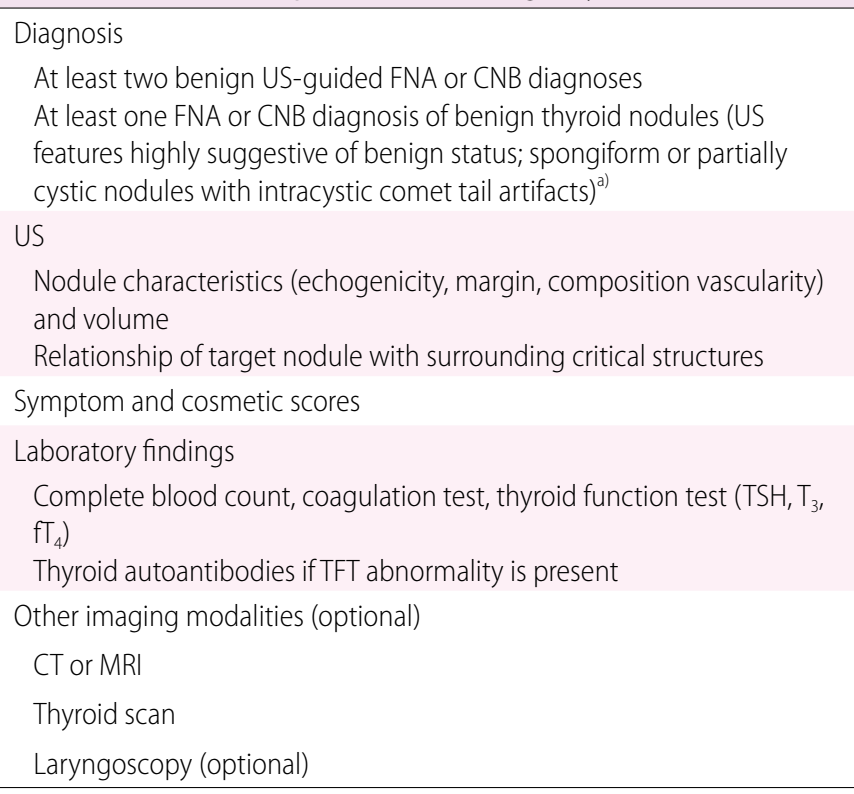

RFA, radiofrequency ablation; US, ultrasound; FNA, fine-needle aspiration; CNB, core needle biopsy; TSH, thyroid-stimulating hormone; TFT, thyroid function test; CT, computed tomography; MRI, magnetic resonance imaging; ACR, American College of Radiology; EU-TIRADS, European Thyroid Imaging Reporting and Data System; AACE/ACE-AME, American Association of Clinical Endocrinologists/American College of Endocrinology and Associazione Medici Endocrinologi; K-TIRADS, Korean Society of Thyroid Radiology Thyroid Imaging Reporting and Data System.

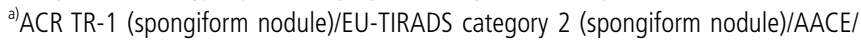
ACE-AME low-risk (spongiform, mostly cystic with intracystic comet tail artifacts)/ K-TIRADS category 2 (spongiform or partially cystic with intracystic comet tail artifacts) nodules. 
thyroid function can be considered to indicate successful ablation. Autoantibodies (anti-thyroid peroxidase and anti-thyroglobulin antibodies) can be selectively measured in some cases. Computed tomography or magnetic resonance imaging may be useful to assess nodules for suspected intrathoracic extension and tracheal compression. A laryngoscopic examination to evaluate vocal cord status is not routinely recommended prior to RFA. However, a laryngoscopic examination is necessary for patients with suspected vocal cord paralysis. US evaluation of the larynx can be effective for the patients with no history of hoarseness. Any prescribed medications that can cause bleeding should be discontinued prior to RFA (7-10 days for aspirin or clopidogrel, 3-5 days for warfarin, and 4-6 hours for heparin). Patients can resume their medication after RFA (2-6 hours for heparin, the following night for warfarin, and the following day for aspirin or clopidogrel) [29].

\section{Procedure}

\section{Key question 4: What is the most appropriate technique for RFA of benign thyroid nodules? Recommendation 4.1}

Perithyroidal lidocaine injection is recommended for local anesthesia.

We recommend a perithyroidal lidocaine injection to reduce pain during the procedure, as sensory nerves are usually present along the thyroid capsule. However, a direct injection of lidocaine into the region of the recurrent laryngeal nerve is not recommended during the procedure, in order to avoid voice changes [2-6].

\section{Debate and consensus}

Considering the balance between benefits and harms, general anesthesia is not recommended. For example, early detection of major complications, such as nerve damage or tracheal injury, may not be possible under general anesthesia. However, conscious sedation is a potential option for nervous patients or those with special clinical concerns. A recent paper reported that nerve damage could be effectively managed by injecting cold $5 \%$ dextrose water (DW) into the area surrounding the damaged nerve [30]. In cases where conscious sedation is required, RFA should be performed carefully to avoid complications, particularly by sparing areas near the critical structures, including the recurrent laryngeal nerve, cervical sympathetic ganglion, and vagus nerve, that are vulnerable to thermal injury $[10,31,32]$.

\section{Recommendation 4.2}

A trans-isthmic approach and the moving-shot technique are recommended as standard.

The trans-isthmic approach and moving-shot technique are fundamental for safe and effective RFA. In the trans-isthmic approach, the electrode needle is inserted via the thyroid isthmus in the midline-to-lateral direction. This technique has several advantages: first, the entire length of the electrode can be visualized; secondly, exposure of the danger triangle to heat can be minimized; and thirdly, movement of the electrode is minimized during swallowing and talking. After insertion of the electrode using the trans-isthmic approach, RFA is performed using the moving-shot technique. The moving-shot technique was developed specifically for the thyroid gland, as thyroid nodules are ellipsoidal and exophytic to the thyroid gland, but lie close to many critical structures such as the esophagus, trachea, recurrent laryngeal nerve, vagus nerve, cervical sympathetic ganglion, and carotid artery. This technique involves dividing the thyroid nodule into multiple small ablation units, which are then ablated by moving the electrode around. The electrode is inserted at the deepest and most remote portion of the target nodule, and is then moved backward as the needle is gradually withdrawn [33].

Advanced techniques, including vascular ablation and hydrodissection, have recently been introduced for thyroid RFA $[2,34]$. Vascular ablation includes two different techniques: arteryfirst ablation and marginal venous ablation. These techniques can reduce marginal recurrence in cases with hypervascular nodules. Hydrodissection can preserve a safety margin around adjacent critical structures during RFA. In this technique, 5\% DW is injected to separate the thyroid nodules from many critical structures; the nodule margin can then be treated safely using the $5 \%$ DW as a thermal barrier $[2,35,36]$.

\section{Debate and consensus}

Contrast-enhanced US also has some advantages for the complete ablation of nodules and can be applied during the procedure at the operator's discretion [37,38]. However, several clinical issues remain, such as high cost, insurance coverage, and invasiveness.

\section{Postprocedural Evaluation}

Following RFA, all clinical, imaging, and laboratory findings should be re-evaluated. The ultimate goal of RFA is clinical success, which is defined as the resolution of clinical problems (i.e., symptoms and cosmetic issues in patients with nonfunctioning nodules and thyroid function test normalization in patients with AFTN) [39]. We generally recommend that follow-up visits be scheduled for 1, 6, and 12 months, and every 6-12 months thereafter, according to the status of the treated nodule. As the successful treatment of nonfunctioning benign thyroid nodules is dependent on improving symptoms and cosmetic issues by reducing nodule volume, US findings, as well as 
symptom and cosmetic scores, should be evaluated after RFA and compared with previous scores. On follow-up US images, physicians should evaluate the nodule size and volume, echogenicity, and internal vascularity. The volume reduction rate (VRR) can be calculated as follows: (initial volume-final volume) $\times 100 /$ initial volume. Regular thyroid function test follow-up is not routinely recommended after treatment of nonfunctioning benign thyroid nodules; however, it is necessary for patients with clinical symptoms of thyroid function abnormality or US suspicion of diffuse thyroid disease prior to RFA.

Following RFA for AFTNs, thyroid function tests that measure $\mathrm{TSH}_{1} \mathrm{~T}_{3}$, and $\mathrm{fT}_{4}$ should be carried out; thyroid scans can also be helpful to determine the therapeutic response. In patients with elevated thyroid antibodies prior to RFA, careful evaluation of the thyroid function test is necessary, as some patients may exhibit hypothyroidism following treatment.

\section{Key question 5: When should additional treatment be considered after RFA? \\ Recommendation 5}

Additional treatment is recommended when the treated nodule shows marginal regrowth or $<50 \%$ volume reduction, and when there is incomplete resolution (or relapse) of symptoms/cosmetic issues.

\section{Debate and consensus}

The indications for additional RFA have not yet been fully defined. However, we recommend additional RFA in the following scenarios: marginal regrowth of the treated nodule (which is defined as nodule volume increase of $\geq 50 \%$ compared to the minimum recorded volume measured at a given follow-up time point), $<50 \%$ VRR, and incomplete resolution (or relapse) of symptoms or cosmetic issues $[2-4,39]$. When the nodule shows vascularity or a volume increase during follow-up, additional ablation should be carefully considered [40]. Regarding AFTNs, the decision for additional treatment is based on the serum TSH level $[2,3,39]$.

\section{Safety}

\section{Key question 6: Is RFA a safe procedure? Recommendation 6}

RFA is a safe procedure when performed by experienced operators.

Previous guidelines and studies, including meta-analyses, have shown that RFA is a safe procedure for the treatment of benign thyroid nodules and has a low incidence of complications [10,31]. The overall complication rate was reported as $2.11 \%$ (95\% confidence interval $[\mathrm{CI}], 1.15$ to 3.06$)$ and the major complication rate was $1.27 \%(95 \% \mathrm{Cl}, 0.81$ to 1.73$)$ [10]. Previous studies have shown that RFA performed by trained radiologists, using a unified protocol and similar devices, is safe for treating benign thyroid nodules. They defined an experienced operator as one who has successfully completed $>50$ thyroid RFAs [12].

However, complications associated with RFA have been reported, including major complications such as nerve injury (including the recurrent laryngeal nerve, cervical sympathetic ganglion, vagus nerve, and brachial plexus), nodule rupture, and permanent hypothyroidism $[10,31]$. Minor complications may include hematoma, vomiting, skin burn, transient thyrotoxicosis, lidocaine toxicity, hypertension, and pain $[10,31]$. There are no known life-threatening complications, and the sequelae rate was reported to be only $0.21 \%$. A thorough knowledge of neck anatomy and its clinical significance is essential for the safe and effective use of US-guided procedures $[32,41,42]$.

\section{Conclusion}

These are the first thyroid RFA guidelines developed by an international academic society. They are based on an analysis of the similarities and differences among five guidelines from different countries and the expert opinion of the ACTA taskforce members. Unresolved issues in these guidelines require further evaluation and discussion.

ORCID: Eun Ju Ha: https://orcid.org/0000-0002-1234-2919; Jung Hwan Baek: https:// orcid.org/0000-0003-0480-4754; Ying Che: https://orcid.org/0000-0002-00444168; Yi-Hong Chou: https://orcid.org/0000-0002-7418-4514; Nobuhiro Fukunari: https://orcid.org/0000-0002-6000-5191; Ji-hoon Kim: https://orcid.org/0000-00026349-6950; Wei-Che Lin: https://orcid.org/0000-0002-1326-0587; Le Thi My: https:// orcid.org/0000-0002-4325-9712; Dong Gyu Na: https://orcid.org/0000-0001-64221652; Lawrence Han Hwee Quek: https://orcid.org/0000-0002-6945-8111; Ming-Hsun Wu: https://orcid.org/0000-0002-8811-9203; Koichiro Yamakado: https://orcid.org/00000001-9354-1524; Jianhua Zhou: https://orcid.org/0000-0003-2096-8126

\section{Author Contributions}

Conceptualization: Baek JH, Ha EJ. Drafting of the manuscript: Baek $J \mathrm{H}, \mathrm{Ha}$ EJ. Critical revision of the manuscript: Ha EJ, Baek JH, Che Y, Chou YH, Fukunari N, Kim JH, Lin WC, My LT, Na DG, Quek LHH, Wu MH, Yamakado K, Zhou J. Approval of the final version of the manuscript: all authors.

\section{Conflict of Interest}

Dr. Baek has acted as a consultant for two companies, STARmed and RF Medical, since 2017. No other member of the guideline committee has any financial disclosure or conflict of interests to declare. 


\section{References}

1. Gharib H, Hegedus L, Pacella CM, Baek JH, Papini E. Clinical review: nonsurgical, image-guided, minimally invasive therapy for thyroid nodules. J Clin Endocrinol Metab 2013;98:3949-3957.

2. Kim JH, Baek JH, Lim HK, Ahn HS, Baek SM, Choi YJ, et al. 2017 Thyroid radiofrequency ablation guideline: Korean Society of Thyroid Radiology. Korean J Radiol 2018;19:632-655.

3. Papini E, Pacella CM, Solbiati LA, Achille G, Barbaro D, Bernardi $S$, et al. Minimally-invasive treatments for benign thyroid nodules: a Delphi-based consensus statement from the Italian minimallyinvasive treatments of the thyroid (MITT) group. Int J Hyperthermia 2019;36:376-382.

4. Garberoglio R, Aliberti C, Appetecchia M, Attard M, Boccuzzi G, Boraso $F$, et al. Radiofrequency ablation for thyroid nodules: which indications? The first Italian opinion statement. J Ultrasound 2015;18:423-430.

5. Dobnig H, Zechmann W, Hermann M, Lehner M, Heute D, Mirzaei $S$, et al. Radiofrequency ablation of thyroid nodules: "Good Clinical Practice Recommendations" for Austria: an interdisciplinary statement from the following professional associations: Austrian Thyroid Association (OSDG), Austrian Society for Nuclear Medicine and Molecular Imaging (OGNMB), Austrian Society for Endocrinology and Metabolism (OGES), Surgical Endocrinology Working Group (ACE) of the Austrian Surgical Society (OEGCH). Wien Med Wochenschr 2020;170:6-14.

6. National Institute for Health and Care Excellence. Ultrasoundguided percutaneous radiogrequency ablation for benign thyroid nodules [Internet]. London: National Institute for Health and Care Excellence, 2016 [cited 2020 Jul 24]. Available from: https:// www.nice.org.uk/guidance/ipg562.

7. Che $Y$, Jin S, Shi C, Wang L, Zhang $X$, Li Y, et al. Treatment of benign thyroid nodules: comparison of surgery with radiofrequency ablation. AJNR Am J Neuroradiol 2015;36:1321-1325.

8. Deandrea M, Sung JY, Limone P, Mormile A, Garino F, Ragazzoni $F$, et al. Efficacy and safety of radiofrequency ablation versus observation for nonfunctioning benign thyroid nodules: a randomized controlled international collaborative trial. Thyroid 2015;25:890-896.

9. Ha EJ, Baek JH, Kim KW, Pyo J, Lee JH, Baek SH, et al. Comparative efficacy of radiofrequency and laser ablation for the treatment of benign thyroid nodules: systematic review including traditional pooling and bayesian network meta-analysis. J Clin Endocrinol Metab 2015;100:1903-1911.

10. Chung SR, Suh CH, Baek JH, Park HS, Choi YJ, Lee JH. Safety of radiofrequency ablation of benign thyroid nodules and recurrent thyroid cancers: a systematic review and meta-analysis. Int J Hyperthermia 2017;33:920-930.

11. Cesareo R, Palermo A, Benvenuto D, Cella E, Pasqualini V, Bernardi S, et al. Efficacy of radiofrequency ablation in autonomous functioning thyroid nodules: a systematic review and meta-analysis. Rev Endocr Metab Disord 2019;20:37-44.

12. Jung SL, Baek JH, Lee JH, Shong YK, Sung JY, Kim KS, et al. Efficacy and safety of radiofrequency ablation for benign thyroid nodules: a prospective multicenter study. Korean J Radiol 2018;19:167-174.

13. Lee M, Baek JH, Suh CH, Chung SR, Choi YJ, Lee JH, et al. Clinical practice guidelines for radiofrequency ablation of benign thyroid nodules: a systematic review. Ultrasonography 2020 Jun 8 [Epub]. https://doi.org/10.14366/usg.20015.

14. Kim JH, Baek JH, Lim HK, Na DG. Summary of the 2017 thyroid radiofrequency ablation guideline and comparison with the 2012 guideline. Ultrasonography 2019;38:125-134.

15. Haugen BR, Alexander EK, Bible KC, Doherty GM, Mandel SJ, Nikiforov YE, et al. 2015 American Thyroid Association management guidelines for adult patients with thyroid nodules and differentiated thyroid cancer: the American Thyroid Association Guidelines Task Force on Thyroid Nodules and Differentiated Thyroid Cancer. Thyroid 2016:26:1-133.

16. Andrews J, Guyatt $G$, Oxman AD, Alderson P, Dahm P, FalckYtter $Y$, et al. GRADE guidelines: 14. Going from evidence to recommendations: the significance and presentation of recommendations. J Clin Epidemiol 2013;66:719-725.

17. Choi SJ, Jeong WK, Jo AJ, Choi JA, Kim MJ, Lee M, et al Methodology for developing evidence-based clinical imaging guidelines: joint recommendations by Korean Society of Radiology and National Evidence-Based Healthcare Collaborating Agency. Korean J Radiol 2017;18:208-216.

18. Dobnig H, Amrein K. Value of monopolar and bipolar radiofrequency ablation for the treatment of benign thyroid nodules. Best Pract Res Clin Endocrinol Metab 2019;33:101283.

19. Li $X L$, Xu HX, Lu F, Yue WW, Sun LP, Bo XW, et al. Treatment efficacy and safety of ultrasound-guided percutaneous bipolar radiofrequency ablation for benign thyroid nodules. $\mathrm{Br} J$ Radiol 2016;89:20150858.

20. Korkusuz Y, Mader A, Groner D, Ahmad S, Mader OM, Grunwald F, et al. Comparison of mono- and bipolar radiofrequency ablation in benign thyroid disease. World I Surg 2017;41:2530-2537.

21. Sung JY, Baek JH, Jung SL, Kim JH, Kim KS, Lee D, et al. Radiofrequency ablation for autonomously functioning thyroid nodules: a multicenter study. Thyroid 2015;25:112-117.

22. Baek JH, Jeong HJ, Kim YS, Kwak MS, Lee D. Radiofrequency ablation for an autonomously functioning thyroid nodule. Thyroid 2008; 18:675-676.

23. Baek JH, Moon WJ, Kim YS, Lee JH, Lee D. Radiofrequency ablation for the treatment of autonomously functioning thyroid nodules. World I Surg 2009;33:1971-1977.

24. Kim HJ, Cho SJ, Baek JH, Suh CH. Efficacy and safety of thermal ablation for autonomously functioning thyroid nodules: a systematic 
review and meta-analysis. Eur Radiol 2020 Aug 20 [Epub]. https:// doi.org/10.1007/s00330-020-07166-0.

25. Tessler FN, Middleton WD, Grant EG, Hoang JK, Berland LL, Teefey SA, et al. ACR Thyroid Imaging, Reporting and Data System (TIRADS): white paper of the ACR TI-RADS Committee. J Am Coll Radiol 2017; 14:587-595.

26. Gharib H, Papini E, Garber JR, Duick DS, Harrell RM, Hegedus L, et al. American Association of Clinical Endocrinologists, American College of Endocrinology, and Associazione Medici Endocrinologi medical guidelines for clinical practice for the diagnosis and management of thyroid nodules: 2016 update. Endocr Pract 2016;22:622-639.

27. Shin JH, Baek JH, Chung J, Ha EJ, Kim JH, Lee YH, et al. Ultrasonography diagnosis and imaging-based management of thyroid nodules: revised Korean Society of Thyroid Radiology consensus statement and recommendations. Korean J Radiol 2016:17:370-395.

28. Russ G, Bonnema SJ, Erdogan MF, Durante C, Ngu R, Leenhardt L. European Thyroid Association guidelines for ultrasound malignancy risk stratification of thyroid nodules in adults: the EU-TIRADS. Eur Thyroid J 2017;6:225-237.

29. Kwok A, Faigel DO. Management of anticoagulation before and after gastrointestinal endoscopy. Am J Gastroenterol 2009; 104:3085-3097.

30. Chung SR, Baek JH, Choi YJ, Lee JH. Management strategy for nerve damage during radiofrequency ablation of thyroid nodules. Int J Hyperthermia 2019:36:204-210.

31. Baek JH, Lee JH, Sung JY, Bae JI, Kim KT, Sim J, et al. Complications encountered in the treatment of benign thyroid nodules with USguided radiofrequency ablation: a multicenter study. Radiology 2012;262:335-342.

32. Ha EJ, Baek JH, Lee JH. Ultrasonography-based thyroidal and perithyroidal anatomy and its clinical significance. Korean J Radiol 2015;16:749-766.
33. Ha EJ, Baek JH, Lee JH. Moving-shot versus fixed electrode techniques for radiofrequency ablation: comparison in an ex-vivo bovine liver tissue model. Korean J Radiol 2014;15:836-843.

34. Park HS, Baek JH, Park AW, Chung SR, Choi YJ, Lee JH. Thyroid radiofrequency ablation: updates on innovative devices and techniques. Korean J Radiol 2017;18:615-623.

35. Cui D, Ding M, Tang $X$, Chi J, Shi Y, Wang T, et al. Efficacy and safety of a combination of hydrodissection and radiofrequency ablation therapy for benign thyroid nodules larger than $2 \mathrm{~cm}$ : a retrospective study. J Cancer Res Ther 2019;15:386-393.

36. Xiaoyin T, Ping L, Dan C, Min D, Jiachang C, Tao W, et al. Risk assessment and hydrodissection technique for radiofrequency ablation of thyroid benign nodules. J Cancer 2018;9:3058-3066.

37. Cervelli R, Mazzeo S, De Napoli L, Boccuzzi A, Pontillo-Contillo B, Materazzi $G$, et al. Radiofrequency ablation in the treatment of benign thyroid nodules: an efficient and safe alternative to surgery. J Vasc Interv Radiol 2017;28:1400-1408.

38. Zhang M, Luo $Y$, Zhang $Y$, Tang J. Efficacy and Safety of Ultrasound-guided radiofrequency ablation for treating low-risk papillary thyroid microcarcinoma: a prospective study. Thyroid 2016;26:1581-1587.

39. Mauri G, Pacella CM, Papini E, Solbiati L, Goldberg SN, Ahmed M, et al. Image-guided thyroid ablation: proposal for standardization of terminology and reporting criteria. Thyroid 2019;29:611-618.

40. Sim JS, Baek JH. Long-term outcomes following thermal ablation of benign thyroid nodules as an alternative to surgery: the importance of controlling regrowth. Endocrinol Metab (Seoul) 2019;34:117123.

41. Mauri G, Nicosia L, Della Vigna P, Varano GM, Maiettini D, Bonomo $G$, et al. Percutaneous laser ablation for benign and malignant thyroid diseases. Ultrasonography 2019:38:25-36.

42. Kotewall N, Lang BHH. High-intensity focused ultrasound ablation as a treatment for benign thyroid diseases: the present and future. Ultrasonography 2019;38:135-142. 